\title{
Switchable Erbium Doped Fiber Ring Laser System for Temperature Sensors Multiplexing
}

\author{
Sergio Rota-Rodrigo, Luis Rodríguez-Cobo, María A. Quintela, Jose M. López-Higuera, Senior \\ Member, IEEE and Manuel López-Amo Senior Member, IEEE
}

\begin{abstract}
In this paper a proof of concept of a switchable erbium doped fiber ring laser (EDFRL) system, demonstrated for temperature sensors multiplexing, was carried out showing a high linearity and a sensitivity of $1.55 \mathrm{GHz} /{ }^{\circ} \mathrm{C}$. The system is based on the detection in the electrical domain of the resulting beat signal of two different laser lines generated by an EDFRL that operates in single longitudinal mode regime (SLM). The SLM operation is achieved by the use of phase shift fiber Bragg gratings (PSFBGs) with ultra-narrow bandwidths (BW) of 6 pm. Two Independent cavities for the reference signal and sensor signal generation are used to avoid the wavelength competition and to improve the stability. This configuration allows the control of the temperature operation point of the system by adjusting the temperature in the reference side.
\end{abstract}

Index Terms-Erbium-doped fiber amplifier, Fiber lasers, Optical sensors, Temperature sensors.

\section{INTRODUCTION}

$\mathrm{O}$ PTICAL fiber sensors can provide attractive means to monitor a variety of external perturbations as temperature, strain, bend, pressure, etc... Due to their wellknown advantages such as immunity to electromagnetic interferences and high sensitivity, they have received an increasing attention in recent years. Within the fiber optic sensors, one of the most extended system are the based on fiber Bragg gratings (FBGs). These kinds of sensors are widely used in structural health monitoring due to the high linearity in their response with temperature and strain. Also FBG technology has the added benefits of a mechanical robustness in harsh environments, compactness and simplicity in fabrication [1-3]. High resolution FBG technology has been also tested in biomedical applications [4] or in the monitoring of the manufacturing process [5]. There are different multiplexing methods for FBG sensors. One of the most

Manuscript received December 12, 2012. The authors are grateful to the Spanish government project TEC2010-20224-C02, to the Spanish Ministry of Education and Culture, and to the grant AP2009-1403.

S. Rota-Rodrigo and M. López-Amo are with the Department of Electronic and Electronic Engineering, Universidad Pública de Navarra, Campus Arrosadia s/n E-31006 Pamplona, Spain. (corresponding author e-mail: Sergio.rota@unavarra.es)

L. Rodríguez-Cobo, M.A. Quintela and J.M. López-Higuera, are with the Photonics Engineering Group, Universidad de Cantabria, Av. Los Castros s/n, 39005, Santander, Spain. extended is the use of erbium doped fiber lasers based on FBGs reflectors for monitoring their wavelength variations into the optical domain [6-8]. On the other hand, several developed sensors use the electrical domain for their interrogation trough the frequency beating technique. These kinds of systems have a high sensitivity response with temperature changes and therefore a very small wavelength variation that cannot be measured using wavelength detectors. In [9] a temperature sensor based on the beating of a dualwavelength laser was demonstrated. The limitation of this system was the measured range due to the direct dependence of this range with the BW of the detector. In [10] was used a commercial tunable laser source (TLS) for beating its emission with the EDFRL signal. This system presented the same problem in the measurement range and increased the final cost because of the addition of a TLS.

Another important issue is the convenience of using multiplexing techniques in order to reduce the cost per each sensor into the measurement system. A number of methods for sensors multiplexing have been investigated, such as wavelength division multiplexing [11] or time division multiplexing [12].

Multi-wavelength erbium doped fiber ring lasers (MEDFRL) are very attractive sources, due to their advantages: they are simple structures that show a narrow linewidth and compatibility with other optical fiber components $[1,13]$. However, ring lasers are also susceptible to output power instabilities that can degrade the performance characteristics of the sensor. Different techniques have been proposed to decrease the instability; for instance, by cooling the erbium doped fiber (EDF)[14], using a dedicated EDF for each wavelength [15], or combining the erbium doped fiber amplifiers (EDFA) with semiconductor optical amplifiers (SOA) [16] or with Raman amplification [17].

In this paper a proof of concept of a switchable optical fiber laser system for the multiplexing of temperature sensors has been carried out. It is based on the electrical detection of the beat of two EDFRL emission lines in single longitudinal mode operation. The SLM behavior is achieved by using PSFBGs with ultra-narrow BW. We used two independent rings for EDFRLs in order to reduce the output power competition and improve the stability. This configuration allows us to extend the temperature measurement range by using a temperature control reference. 


\section{EXPERIMENTAL SETUP}

Figure 1 shows the experimental setup of the proposed temperature-sensor system. That is based on the electrical domain detection of the mixing of two single-longitudinal mode erbium-doped fiber-ring laser emission lines.

The wavelength selection of both emission lines obtained from our EDFRL: reference line and sensors lasing signal, is carried out using a combination of FBGs and PSFBGs .The FBG is located in-reflection using a circulator and its purpose is the coarse wavelength selection. The FBGs are centered on $1550 \mathrm{~nm}$, measured at room temperature $\left(24^{\circ} \mathrm{C}\right)$, having a reflectivity close to $90 \%$ and a corresponding full-width at half maximum (FWHM) of $0.120 \mathrm{~nm}$. The PSFBG actuated as a narrow transmission filter with a FWHM less than $6 \mathrm{pm}$.

Based on [18], any phase shift can be obtained by changing the sampling period of a Sampled Fiber Bragg Grating (SFBG) [19]. For this application the PSFBGs were written into a standard telecommunication optical fiber using the phase mask technique illuminated with a continuous laser emitting at 244 $\mathrm{nm}$. The sampling effect was generated by opening and closing the laser shutter at preset locations. The total length of the PSFBGs was $L \approx 24 \mathrm{~mm}$ with a sampling period of $\mathrm{P}=1.5 \mathrm{~mm}$. An increment in the sampling period $\mathrm{P}$ with a scale factor of $\mathrm{F}=1.5$ located in the center of the grating creates an equivalent pi-phase shift in the first order of the SFBG. The obtained spectrum of the phase-shifted SFBG is detailed in the Figure 2.

The utilized gain medium into the laser consists of two EDFs of $7 \mathrm{~m}$, one for the reference EDFRL and the other for the lasing sensor arm. These EDFs are pumped with the same laser at $1480 \mathrm{~nm}$ trough the 50:50 optical coupler, with a pump power of $200 \mathrm{mw}$. The selection of each sensor is made by an optical switch (HP 86061C) with a maximum switching time of $370 \mathrm{~ms}$. The four outputs of the switch are connected to the sensing elements that consist of couples of FBG and PSFBG.
A $4 \times 2$ coupler collects the signal from the operational branch of the switch. The isolators ensures the unidirectional operation of each EDFRL and therefore avoids the spatial hole-burning effect.

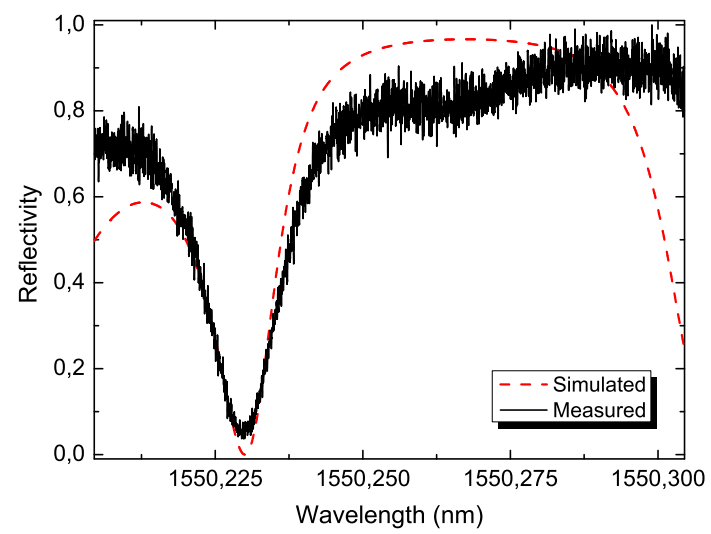

Fig. 2. Simulated and measured in reflection spectra for the phase shift of the SFBG first order

The signals from the selected sensor and the reference are mixed by a 50:50 coupler and their outputs are converted to the electrical domain by the photodetector (HP 11982A) that shows a BW of $20 \mathrm{GHz}$. An electrical spectrum analyzer (ESA) with $1 \mathrm{KHz}$ of resolution (model HP 8592L) is used to retrieve the output signal.

For the sensor characterization a Peltier unit having a thermo electrical cooler (TEC) is used. Figure 3 shows the disposition of the FBG and PSFBG on the Peltier. Each couple of FBG and PSFBG experiments the same temperature change and therefore the same variation at their wavelengths. A conducting paste was used to ensure the uniform distribution of the temperature.

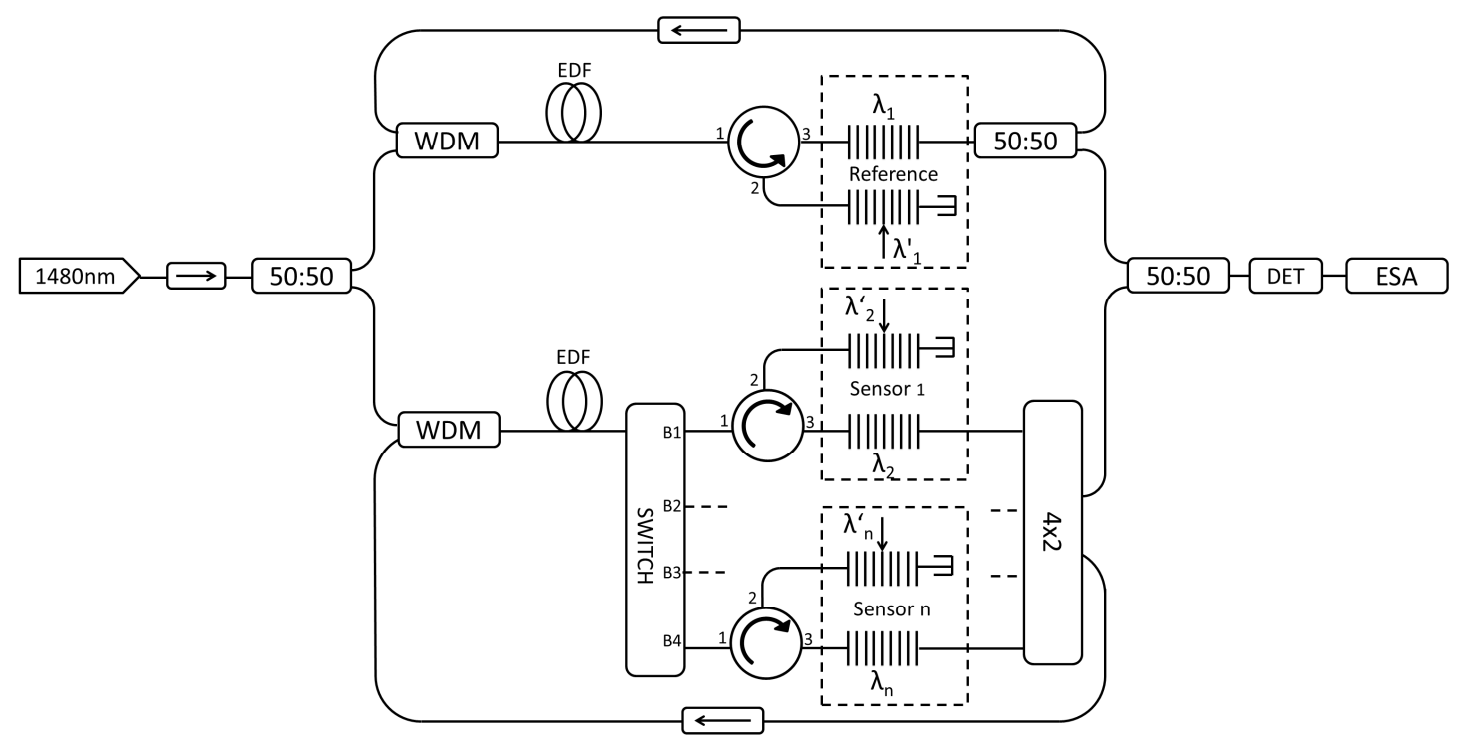

Fig. 1. Experimental setup of the proposed switchable EDFRL system for temperature sensors multiplexing, where $\lambda_{\mathrm{i}}$ and $\lambda_{\mathrm{i}}$ ' are the FBGs and the PSFBGs, respectively. 


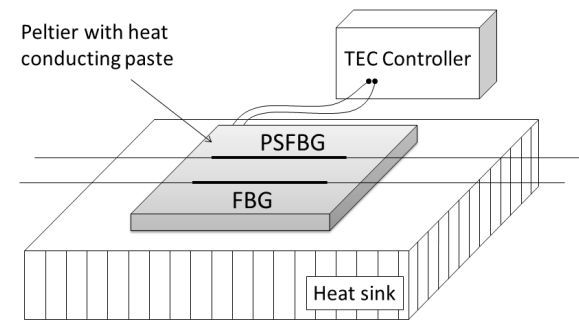

Fig. 3. Schematic representation of the experimental setup for the sensors temperature test.

\section{EXPERIMENTAL RESULTS}

Figure 4a shows the optical spectrum at $24^{\circ} \mathrm{C}$ measured at a high-resolution Brillouin optical spectrum analyzer (BOSAC Aragon Photonics) with a resolution of $0.08 \mathrm{pm}$. The wavelengths correspond to the reference EDFRL and the EDFRL of sensor 1 . Both lasing signals operate in single longitudinal mode regime. Figure $4 \mathrm{~b}$ displays the electrical spectra of the beat of these signals demonstrating the SLM behavior of both EDFRL. The peak at $11.9 \mathrm{GHz}$ corresponds to the spacing between both lasers.
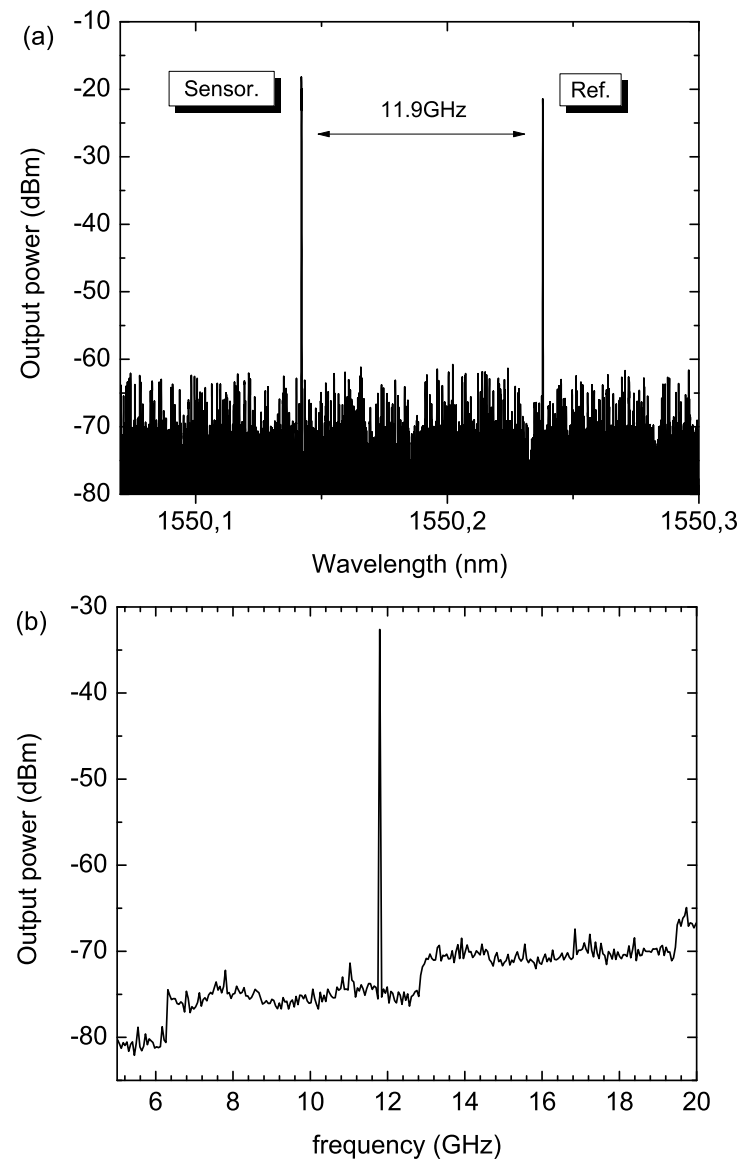

Fig. 4. Optical output spectrum at $24^{\circ} \mathrm{C}$ measured at BOSA (a) and electrical output spectrum at $24^{\circ} \mathrm{C}$ measured at ESA (b).
In order to determinate the response of the sensor with temperature variations and its instability, a series of measurements for sensor 1 , ranging from $19{ }^{\circ} \mathrm{C}$ to $30{ }^{\circ} \mathrm{C}$ with steps of $1{ }^{\circ} \mathrm{C}$ was carried out. The reference arm operated at room temperature $\left(24{ }^{\circ} \mathrm{C}\right)$. Each measurement step consisted of a period of 10 minutes with captures every minute (Fig 5a). The frequency instability was lower than $200 \mathrm{MHz}$, value that as can be seen below not affected significantly the linearity of the sensor. This instability was due to the TEC instability in combination with the thermal insulation tolerance of the characterization setup that can easily cause fluctuations of $0.1{ }^{\circ} \mathrm{C}$. Figure $5 \mathrm{~b}$ shows the beat frequency dependence with the temperature, measured by using a time averaging technique. High sensitivity of $1.55 \mathrm{GHz} /{ }^{\circ} \mathrm{C}$ with a linearity of 0.9993 was obtained, being these values are better than the obtained in [10], that were $1.30 \mathrm{GHz} /{ }^{\circ} \mathrm{C}$ with a linearity of 0.9662 or in [9] $1.47 \mathrm{GHz} /{ }^{\circ} \mathrm{C}$ with a linearity of 0.9935 .
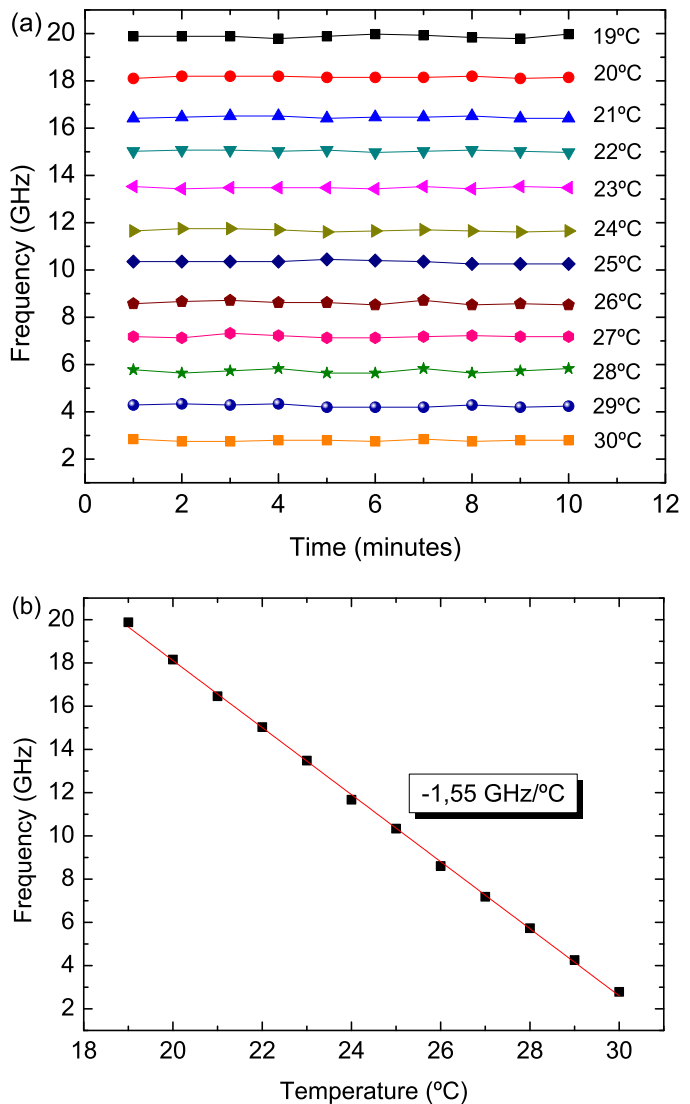

Fig. 5. Beat frequency dependence with time at different temperatures (a). Average beat frequency shift dependence with temperature (b).

Considering that all of the FBGs and PSFBGs were written in the same fiber and with the same process, all of them have the same behavior with the temperature $\left(1.55 \mathrm{GHz} /{ }^{\circ} \mathrm{C}\right)$ resulting in an absolute equation for the measured temperature:

$$
T\left({ }^{\mathrm{o}} \mathrm{C}\right)=-\frac{\operatorname{Freq}(G H z)-49.122}{1.55}+\left(T_{r e f}-24\right)
$$


By controlling the reference temperature (Tref) with a Peltier system we can displace the temperature operation point of the system maintaining the BW of the detector. Figure 6 shows the theoretical range of an application where different reference temperatures are employed to improve the dynamic range. This is an important advantage in comparison with other sensors systems $[9,10]$ wherein the temperature range is limited by the $\mathrm{BW}$ of the detector.

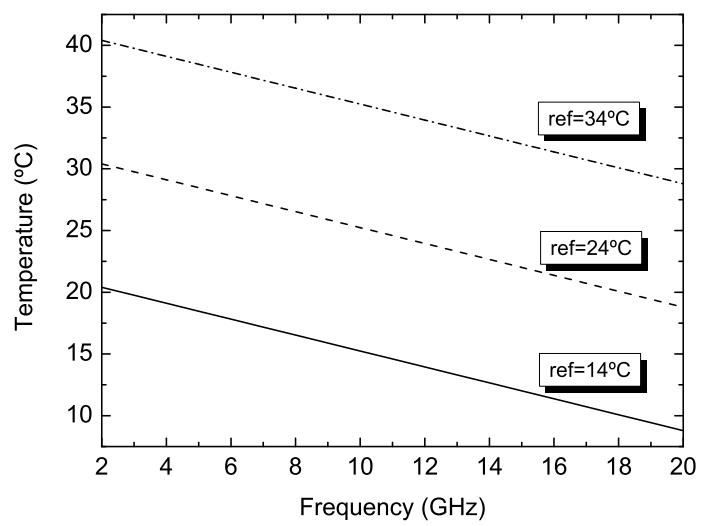

Fig. 6. Theoretical beat frequency shift dependence with temperature for different reference temperatures.

The transient switching response of the sensors was also studied. This switching time is defined as the time delay between the moment in which one sensor is turned off and the other has a stabilized output. For the measurement we employed a photo-detector (PDA400 provide by Thorlabs) and an oscilloscope (Agilent 54622A). Figure 7 shows the switching measured time from B1 to B4 output (solid line) and from B4 to B1 output (dotted line). For both the switching time were less than $250 \mathrm{~ms}$.

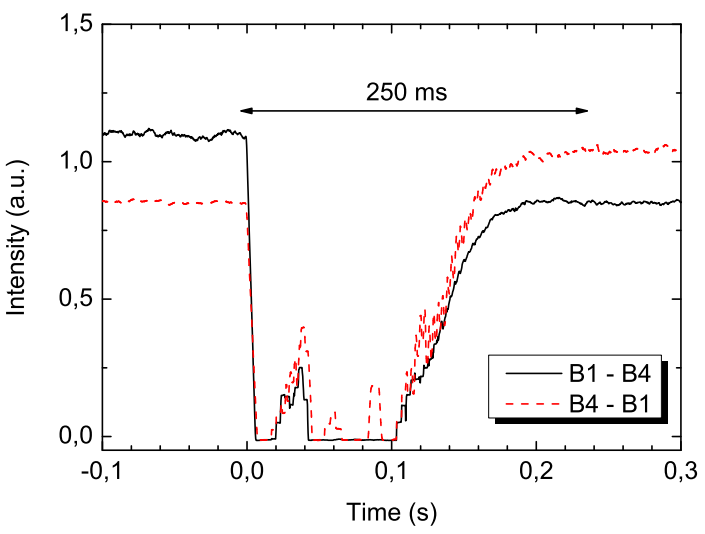

Fig. 7. Switching time from B1 to B4 output (solid line) and from B4 to B1 output (dotted line).

\section{CONCLUSIONS}

In conclusion, a new switchable erbium doped fiber ring laser system for multiplexing temperature sensors was proposed and experimentally demonstrated. The measurements show a high sensitivity of $1.55 \mathrm{GHz} /{ }^{\circ} \mathrm{C}$ and a high linearity. The system is based on the electrical domain detection of the beat of two laser lines operating in single longitudinal mode regime. The SLM operation is achieved by the use of PSFBGs with ultra-narrow bandwidths. Independent cavities for the generation of the reference signal and the sensors signals were used to avoid the wavelength competition and to improve the stability. By including a temperature control in the reference signal, the system allows a high dynamic range of operating temperature points for the sensor system, maintaining the receptor BW. Another advantage of the proposed structure is the possibility of increase the number of sensors by changing the $2 \times 4$ switch by another having a higher number of outputs.

\section{REFERENCES}

[1] S.T. Vohra, "Optical Fiber Bragg Gratings Applications". In Hand Book of Optical Fibre Sensing Technology, Lopez-Higuera, J.M (Ed), Wiley \& Sons. Cap.23, 475-504 (2002).

[2] J. M. López-Higuera, L. R. Cobo, A. Q. Incera and A. Cobo, "Fiber optic sensors in structural health monitoring," J. Lightwave Technol., vol. 29, pp. 587-608, 2011.

[3] M. Bravo, J. Sáenz, M. Bravo-Navas and M. Lopez-Amo, "Concrete beam bending test monitorization using a high strain fiber optic sensor," J. Lightwave Technol., vol. 30, pp. 1085-1089, 2012.

[4] Y. J. Rao, D. J. Webb, D. A. Jackson, L. Zhang, I. Bennion, "In-fibre Bragg grating temperature sensor system for medical applications," $J$. Lightwave Technol., vol. 15, pp. 779-7985, 1997.

[5] J. Echevarría, C.Jáuregui, A. Quintela, M. A. Rodríguez, R. García, G. Gutiérrez and J. M. López-Higuera, "Concrete Beam Curing Process and Flexural Test with Fiber Bragg Grating based Transducers," Proc. SPIE, vol. 4694, pp. 271-276, 2002.

[6] R. A. Perez-Herrera, A. Ullan, D. Leandro, M. Fernandez-Vallejo, M. A. Quintela, A. Loayssa, J. M. Lopez-Higuera and M. Lopez-Amo, "L-band multiwavelength single-longitudinal mode fiber laser for sensing applications," J. Lightwave Technol., vol. 30, pp. 1173-1177, 2012.

[7] Q. Han and Z. Sheng, "Tunable erbium-doped fiber ring laser based on a novel thermal tuning scheme of fiber Bragg gratings," Modern Physics Letters B, vol. 24, pp. 963-969, 2010.

[8] O. Hadeler, E. Rønnekleiv, M. Ibsen, and R.I. Laming, "Polarimetric distributed feedback fiber laser sensor for simultaneous strain and temperature measurements" Applied Optics, vol. 38, pp. 1953-1958, 1999.

[9] Y. Dai, Q. Sun, J. Zhang, J. Wo, and D. Liu, "Tunable dual-wavelength double-ring fiber laser and its application in highly sensitive temperature sensing," Lasers and Electro-Optics (CLEO), 2012 conference on, pp 1-2, 2012.

[10] H. Ahmad, A. A. Latif, M. Z. Zulkifli, N. A. Awang and S. W. Harun, "Temperature sensing using frequency beating technique from singlelongitudinal mode fiber laser," IEEE Sensors Journal, vol. 12, pp. 24962500, 2012.

[11] S. Diaz, S. Abad and M. Lopez-Amo, "Fiber-optic sensor active networking with distributed erbium-doped fiber and Raman amplification," Laser and Photonics Reviews, vol. 2, pp. 480-497, 2008.

[12] M. Bravo, J. M. Baptista, J.L. Santos, M. Lopez-Amo and O. Frazão, "Ultralong $250 \mathrm{~km}$ Remote Sensor System Based on a Fiber Loop Mirror Interrogated by an Optical Time-Domain Reflectometer." Opt. Lett. 2011, 36, 4059-4061.

[13] A. Bellemare, "Continuous-wave silica-based erbium-doped fibre lasers," Progress in Quantum Electronics 27, 211-266 (2003).

[14] H. L. An, X. Z. Lin, E. Y. B. Pun and H. D. Liu, "Multi-wavelength operation of an erbium-doped fiber ring laser using a dual-pass MachZehnder comb filter," Opt. Commun., vol. 169, pp. 159-165, 1999.

[15] M. Fernandez-Vallejo, R. A. Perez-Herrera, C. Elosúa, C. Bariain and M. Lopez-Amo, "Stable multiwavelength fiber laser for referencing intensity sensor networks using multiple amplified ring resonators," in Proc. SPIE 7653, Fourth European Workshop on Optical Fibre Sensors, Porto, 2010. 
[16] S. Shahi, S. W. Harun, A. H. Sulaiman, K. Thambiratnam and H. Ahmad, "Multiwavelength source based on SOA and EDFA in a ringcavity resonator," Microwave Opt Technol Lett, vol. 51, pp. 110-113, 2009.

[17] H. Masuda and S. Kawai, "Wide-band and gain-flattened hybrid fiber amplifier consisting of an EDFA and a multiwavelength pumped Raman amplifier," IEEE Photonics Technology Letters, vol. 11, pp. 647-649, 1999.

[18] Y. Dai, X. Chen, D. Jiang, S. Xie and C. Fan, "Equivalent phase shift in a fiber Bragg grating achieved by changing the sampling period," IEEE Photonics Technology Letters, vol. 16, pp. 2284-2286, 2004.

[19] B. J. Eggleton, P. A. Krug, L. Poladian and F. Ouellette, "Long periodic superstructure Bragg gratings in optical fibres," Electron. Lett., vol. 30 , pp. $1620-1622,1994$.

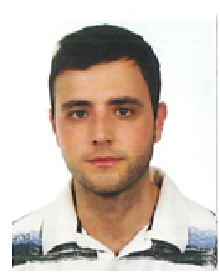

Sergio Rota-Rodrigo was born in Tudela, Navarra, Spain, in October 1985. He received the Telecommunication Technical Engineering, Telecommunication Engineering and Communications Master degrees from the Universidad Pública de Navarra, Spain in 2007, 2010 and 2011, respectively. In 2011, he joined the Optical Communications Group, Department of Electrical and Electronic Engineering from Universidad Pública de Navarra. His research interests are in fiber optic lasers, optical amplifiers, optical fiber sensor networks and chemical fiber optic sensors.

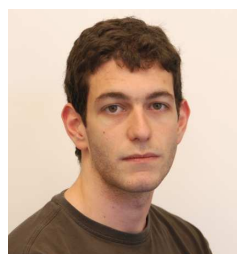

Luis Rodriguez-Cobo received his degree in Telecommunication Engineering from the University of Cantabria (Spain), in 2009. In 2009 he started his $\mathrm{PhD}$ studies at the Photonics Engineering Group (GIF) within the Department of electronic Technology Industrial Automation and System Engineering at the University of Cantabria. He also obtained the TICRM Information Technology and Communications in Mobile Networks) interuniversity Master degree in October of 2010. His research is about applying optical technologies to structural health monitoring systems in renewable energies field.

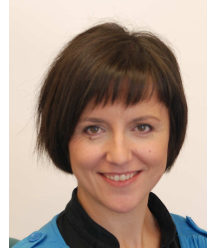

María Angeles Quintela was born in Santander, Spain, in 1974. She received both his Telecommunications Technical Engineering degree and his Telecommunications Engineering degree at the University of Cantabria. In 1998, she joined the University of Cantabria as a lecturer. Her teaching topics include electronic components and optical communications. In 2005 she obtained her Ph.D. degree. Her main research is on computer modelling and experimental characterisation of fiber lasers. She has co-authored more than 60 papers, presented in conferences and scientific journals.

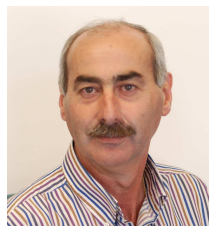

José Miguel López-Higuera was born in February 1954, in the village of Ramales de la Victoria, Cantabria (Spain). He obtained his Telecommunication Technical Engineering degree in the Universidad Laboral de Alcalá de Henares, and his Telecommunication Engineering degree in the Universidad Politécnica de Madrid (UPM). $\mathrm{He}$ obtained his $\mathrm{PhD}$ degree in Telecommunication Engineering, with an extraordinary award, in UPM. He founded and is the head of the Photonics Engineering Group of the TEISA Department in the University of Cantabria. Presently, he works in the development of Photonics Instrumentation, photonic/optical fibre sensor systems for civil engineering, electrical power, environmental and smart structures and for optical diagnostics for a wide range of applications. He has directed more than fifty $\mathrm{R} \& \mathrm{D}$ projects and has written or co-written more than four hundred publications in the form of books, chapters of books, papers and conferences, both national and international, and obtained 10 patents. He is the editor and co-author of several books, including i) "Optical Sensors", UC, 1998; ii) the "Handbook of Photonic Sensing Technology",Wiley and Sons, 2002 and iii) he is the co-editor of the book "Engineering a High-Tech Business: Entrepreneurial experiences and Insights", published by SPIE-Press,USA, 2008. Professor López-Higuera is senior member of the IEEE and member of the IEE, SPIE and OSA.

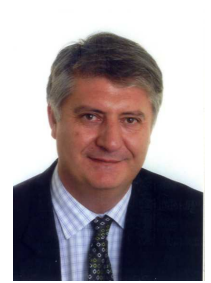

Manuel Lopez-Amo (M'91, SM '98) was born in Madrid, Spain, in 1960. He received the telecommunications engineering degree and Ph.D. degrees from the Universidad Politécnica de Madrid, Spain in 1985 and 1989, respectively. From 1985 to 1996, he belonged to the Photonic Technology Department of the Universidad Politécnica de Madrid., where in 1990 he became an associate professor.

In 1996, he moved to Public University of Navarra (Pamplona, Spain) where he became a Full Professor in the Electrical and Electronic Engineering department and is currently the head of the optical communications group of this department. He has been Chairman of the Optoelectronic Committee of Spain. He has been leader of more than 20 research projects and he has coauthored more than 200 works in international refereed journals and conferences related with fiber-optic networks, optical amplifiers, fiber-optic sensors, and integrated optics. He is a member of the technical committees of the International Conference on fiber optic sensors (OFS), the European Workshop on optical fiber sensors (EWOFS), and European cost TD1001 action, among others. Professor López-Amo is senior member of the IEEE and member of the OSA 